\title{
Study of the nonlinear longitudinal dynamics of a stochastic system
}

\author{
Americo Cunha $\mathrm{Jr}^{\mathrm{a}}$ and Rubens Sampaio ${ }^{\mathrm{b}}$ \\ Department of Mechanical Engineering \\ Pontifícia Universidade Católica do Rio de Janeiro (PUC-Rio) \\ Rua Marquês de São Vicente, 225, Gávea, Rio de Janeiro - RJ, Brazil — 22453-900
}

\begin{abstract}
This paper deals with the theoretical study of how discrete elements attached to a continuous stochastic systems can affect their dynamical behavior. For this, it is studied the nonlinear longitudinal dynamics of an elastic bar, attached to springs and a lumped mass, with a random elastic modulus and subjected to a Gaussian white-noise distributed external force. Numerical simulations are conducted and their results are analyzed in function of the ratio between the masses of the discrete and the continuous parts of the system. This analysis reveals that the dynamic behavior of the bar is significantly altered when the lumped mass is varied, being more influenced by the randomness for small values of the lumped mass.
\end{abstract}

\section{Introduction}

In the context of structural dynamics, it is quite common to model structures as a continuous system coupled with discrete elements. This is the case, for example, of drillstrings [1], carbon nanotubes [2], etc.

These models, like any mathematical model, are subject to uncertainties due to variability of its parameters (physical constants, geometry, etc), and mainly due to the possible inaccuracies committed in the conception of the model (wrong hypotheses about the physics) [3].

In this sense, this work intends to analyze the influence of discrete elements in the longitudinal nonlinear dynamics of a continuous mechanical system subjected to randomness on its parameters. The theoretical study developed aims to illustrate a consistent methodology to analyze the influence of the coupled discrete elements into the stochastic dynamics of nonlinear mechanical systems.

The work is organized as follows. The section 2 presents the physical system of interest, as well as its modeling. In the section 3 , some configurations of the model are analyzed in order to characterize the effect a discrete element in the nonlinear dynamical system. Finally, in the section 4 , the main conclusions are emphasized.

\section{Mathematical modeling}

The physical system of interest in this work, sketched in the Figure 1, consists of an elastic bar which the left side is fixed at a rigid wall, and the right side is attached to a lumped mass and two springs (one linear and one nonlinear). This bar has a length $L$, cross section area $A$, and is made of a material with mass density $\rho$ and elastic modulus $E$. It loses energy through a mechanism of viscous dissipation, with damping coefficient $c$. The stiffness constants of the linear and the nonlinear springs are respectively denoted by $k$ and $k_{N L}$. The lumped mass on the right extreme is $m$.

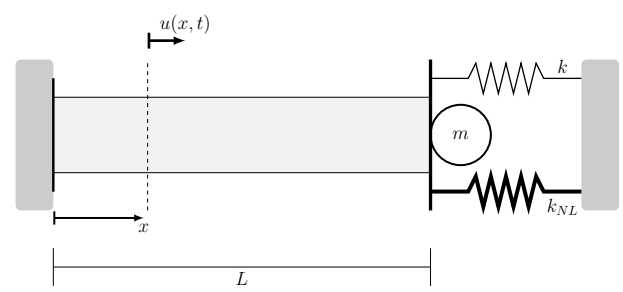

Fig. 1. Sketch of a bar fixed at one and attached to two springs and a lumped mass on the other extreme.

Accordingly, the bar displacement $u(x, t)$ evolves according to

$$
\begin{aligned}
\rho A \frac{\partial^{2} u}{\partial t^{2}}+c \frac{\partial u}{\partial t} & =\frac{\partial}{\partial x}\left(E A \frac{\partial u}{\partial x}\right)+f(x, t) \\
& -\left(k u+k_{N L} u^{3}+m \frac{\partial^{2} u}{\partial t^{2}}\right) \delta(x-L),
\end{aligned}
$$

where the symbol $\delta(x-L)$ denotes the delta of Dirac distribution at $x=L$, and $f$ is a distributed external force, which depends on the position $x$ and time $t$.

\footnotetext{
a e-mail: americo.cunhajr@gmail.com

b e-mail: rsampaio@puc-rio.br
} 
The boundary conditions for this problem are

$$
u(0, t)=0, \text { and } E A \frac{\partial u}{\partial x}(L, t)=0,
$$

while the initial conditions read as

$$
u(x, 0)=u_{0}(x), \text { and } \frac{\partial u}{\partial t}(x, 0)=v_{0}(x),
$$

being $u_{0}$ and $v_{0}$ known functions of $x$.

Using the Galerkin method [4] to construct an approximation to the solution of the boundary/initial value problem above, one gets an initial value problem of the form

$$
\begin{gathered}
{[M] \ddot{\mathbf{u}}(t)+[C] \dot{\mathbf{u}}(t)+[K] \mathbf{u}(t)=\mathbf{f}(t)+\mathbf{f}_{N L}(\dot{\mathbf{u}}(t)),} \\
\mathbf{u}(0)=\mathbf{u}_{0} \quad \text { and } \quad \dot{\mathbf{u}}(0)=\mathbf{v}_{0}
\end{gathered}
$$

which is integrated using a Newmark scheme [4]. In this initial value problem, $\mathbf{u}(t)$ is the vector of unknowns, $[M]$ is the mass matrix, $[C]$ is the damping matrix, $[K]$ is the stiffness matrix. Also, $\mathbf{f}(t), \mathbf{f}_{N L}(\mathbf{u}(t))$, $\mathbf{u}_{0}$, and $\mathbf{v}_{0}$ are vectors which, respectively, represent the external force, the nonlinear force, the initial position, and the initial velocity.

In order to introduce any type of randomness in the physical system, the elastic modulus $E$ is modeled as a random variable which the probability density function (PDF) is gamma. For consistency of modeling, this distribution was specified using the maximum entropy principle [3].

On the other hand, the distributed external force acting on the bar is arbitrarily assumed to be

$$
F(x, t, \theta)=\sigma \phi_{1}(x) N(t, \theta)
$$

where $\sigma$ is the force amplitude, $\phi_{1}$ the first elastic mode of the bar, and $N(t, \theta)$ is a Gaussian white-noise with zero mean and unit variance.

To calculate the propagation of uncertainties of the random parameters through the nonlinear dynamics of the bar, it is used the Monte Carlo method [5,6].

\section{Numerical results}

For the simulations presented in this section, the evolution of the nonlinear dynamical system is investigated for $T=10 \mathrm{~ms}$, and the nominal parameters of Table 1 are adopted. The initial conditions are assumed to be non-zero displacement and zero velocity. Furthermore, the random variable $E$, is characterized by a mean $\mu_{E}=203 G P a$ and a dispersion factor $\delta_{E}=10 \%$.

In the study that follows, it is considered the nominal (deterministic) model, the one which has the parameters of the Table $1, E=\mu_{E}$, and $f(x, t)=\sigma \phi_{1}(x)$.

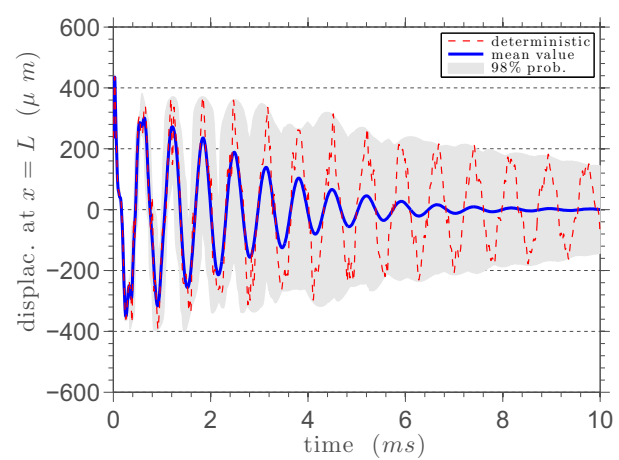

(a) $m^{*}=0.1$

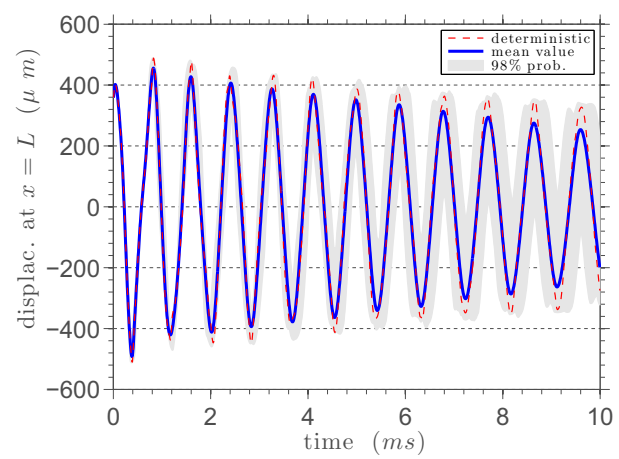

(b) $m^{*}=1$

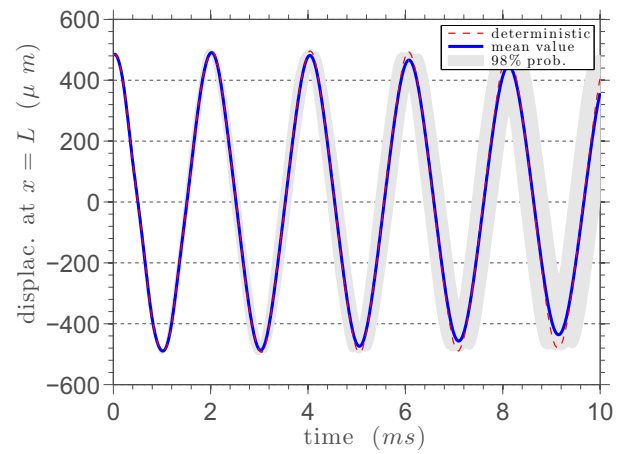

(c) $m^{*}=10$

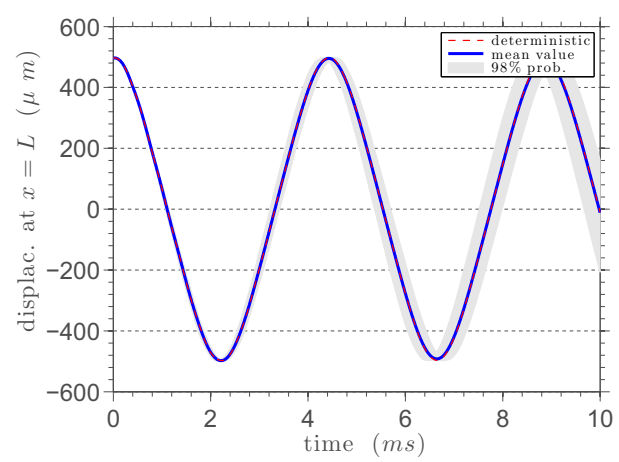

(d) $m^{*}=50$

Fig. 2. This figure illustrates the mean value (blue line), the nominal value (red line), and a $98 \%$ of probability interval of confidence (grey shadow) for the bar right extreme displacement, for several values of $m^{*}$. 
Table 1. Nominal parameters used in the simulations.

\begin{tabular}{lll}
\hline parameter & value & unit \\
\hline$\rho$ & 7900 & $\mathrm{~kg} / \mathrm{m}^{3}$ \\
$A$ & $625 \pi$ & $\mathrm{mm}$ \\
$L$ & 1 & $\mathrm{~m}$ \\
$c$ & 5 & $\mathrm{kN} / \mathrm{s}$ \\
$k$ & 650 & $\mathrm{~N} / \mathrm{m}$ \\
$k_{N L}$ & $650 \times 10^{13}$ & $\mathrm{~N} / \mathrm{m}^{3}$ \\
$\sigma$ & 5 & $\mathrm{kN}$ \\
\hline
\end{tabular}

A parametric study is performed using as the indexing parameter the mass ratio defined by

$$
m^{*}=\frac{m}{\rho A L},
$$

which is the ratio between the discrete and the continuous mass of the physical system. For instance, it is considered $m^{*}=0.1,1,10,50$.

In the Figure 2 one can observe, for different values of $m^{*}$, the mean value of the bar right extreme displacement (blue line), its nominal value (red line), and an envelope of reliability (grey shadow), wherein a realization of the stochastic system has $98 \%$ of probability of being contained. Note that, as the value of lumped mass increases, the mean value tends to the nominal value. One might think that the system is "more random" for small values of $m^{*}$.

The mean trajectory in the system phase space, corresponding to the the bar right extreme, and its nominal value, are shown, for different values of $m^{*}$, in the Figure 3. One can observe a "disturbed" nominal orbit associated $m^{*}=0.1$. The reason for this disturbance is the dependence with the initial conditions, which disappears when $m^{*} \rightarrow \infty$. Also, since the mean orbit tends from a stable focus to an ellipse as $m^{*}$ increases, the system is not much influenced by damping as for large values of $m^{*}$.

In the Figure 4 are presented estimations for the PDFs of the (normalized ${ }^{1}$ ) displacements of the bar right extreme at the instant of time $T$.

In all cases the PDF presents an asymmetry around the mean, which indicates that its more probable the displacement be higher or lower than the mean, according to the area under the PDF curve to the right or to the left of the mean, respectively. Beyond that, it is possible to observe a multimodal PDF for all values of $m^{*}$, which indicates a high number of realizations close to the values that correspond to the peaks. Therefore, the regions near the peaks are areas of greater probability for the system response.

For a deeper discussion on the behavior of this physical system, also taking into account the energy spectrum of the system, the reader is encouraged to see the reference [7].

\footnotetext{
${ }^{1}$ Normalized means a random variable with zero mean and unit standard deviation.
}

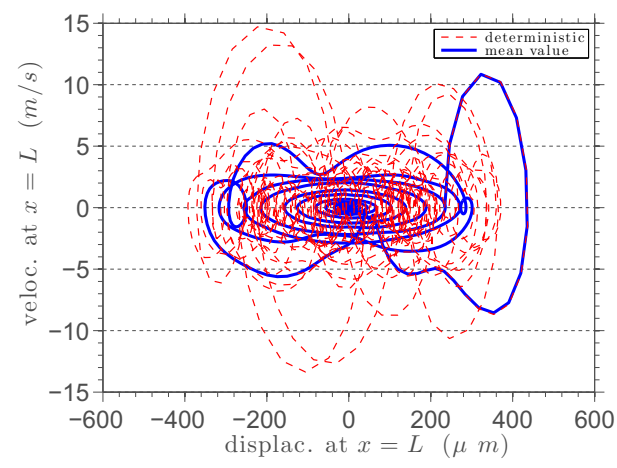

(a) $m^{*}=0.1$

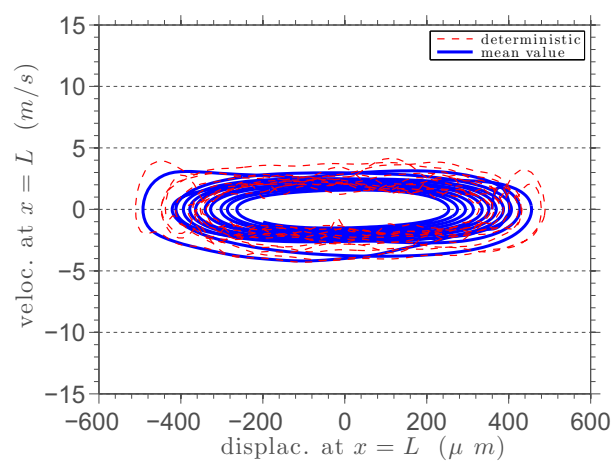

(b) $m^{*}=1$

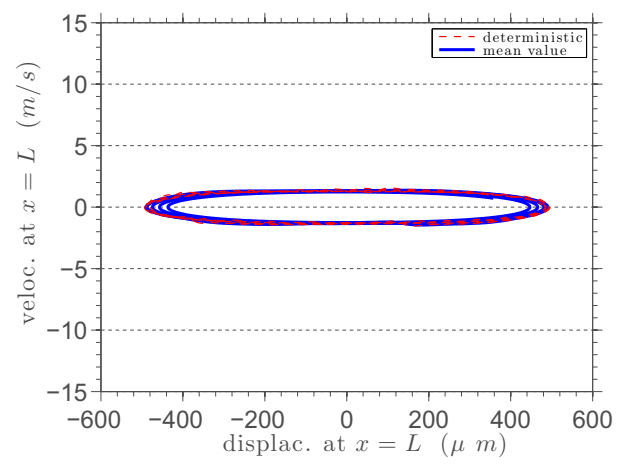

(c) $m^{*}=10$

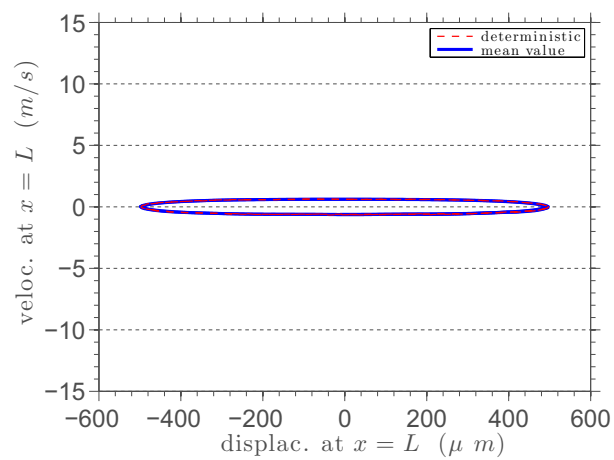

(d) $m^{*}=50$

Fig. 3. This figure illustrates the mean trajectory (blue line) in the system phase space, corresponding to the the bar right extreme, and its nominal value (red line), for several values of $m^{*}$. 


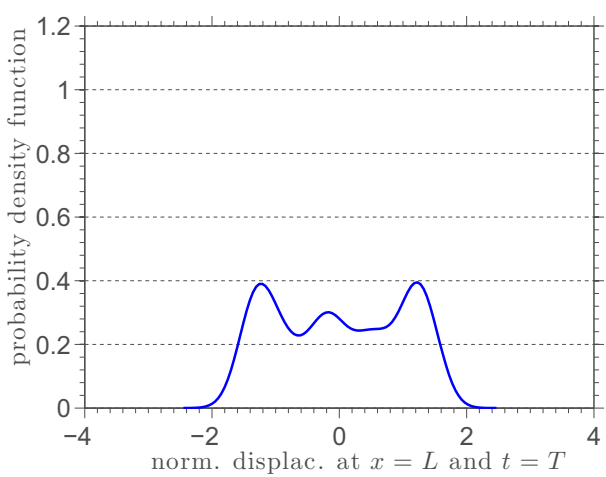

(a) $m^{*}=0.1$

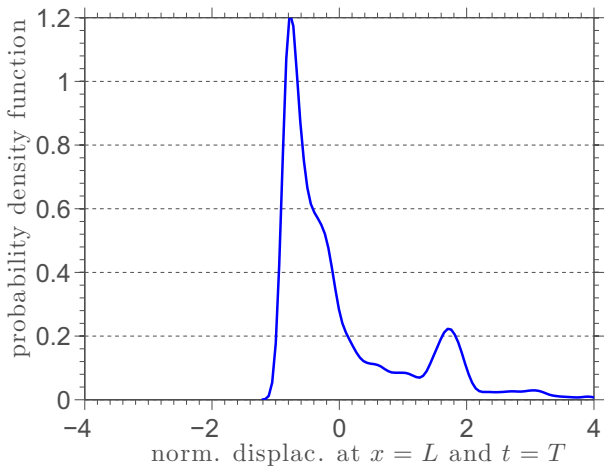

(b) $m^{*}=1$

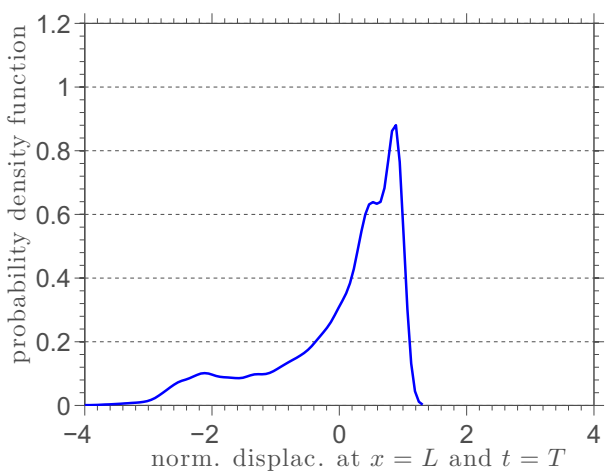

(c) $m^{*}=10$

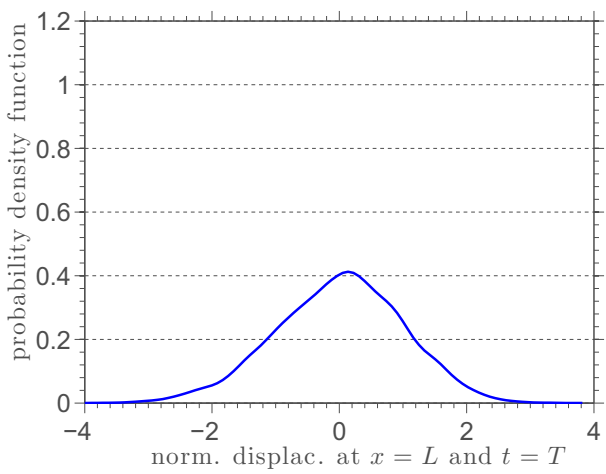

(d) $m^{*}=50$

Fig. 4. This figure illustrates estimations to the PDFs of the (normalized) bar right extreme displacement at the instant of time $T$, for several values of $m^{*}$.

\section{Concluding remarks}

In this work was discussed the longitudinal dynamical behavior of a nonlinear continuous physical system, coupled discrete elements, and subjected to randomness on its parameters. The analysis of the model was performed, indexed by a dimensionless parameter that describes the ratio between the discrete/continuous mass of the system. This analysis shows that the variation of the lumped mass significantly affects the behavior of the system, and this change results in a system that is "more random" for small values of the lumped mass. Furthermore, the probability distributions of bar right extreme displacement present asymmetries and multimodal behavior, so that there are areas greater probability for the dynamic system response.

\section{Acknowledgments}

The authors are indebted to the Brazilian agencies CNPq, CAPES, and FAPERJ for the financial support given to this research.

\section{References}

1. T. G. Ritto, M. R. Escalante, R. Sampaio and M. B. Rosales, Drill-string horizontal dynamics with uncertainty on the frictional force, Journal of Sound and Vibration 332, (2013) 145-153.

2. T. Murmu and S. Adhikari, Nonlocal vibration of carbon nanotubes with attached buckyballs at tip, Mechanics Research Communications 38, (2011) 62-67.

3. C. Soize, Stochastic modeling of uncertainties in computational structural dynamics - Recent theoretical advances, Journal of Sound and Vibration 332, (2013) 2379-2395.

4. T. J. R. Hughes, The Finite Element Method: Linear Static and Dynamic Finite Element Analysis Dover Publications, 2000.

5. C. P. Robert and G. Casella, Monte Carlo Statistical Methods Springer, 2010.

6. A. Cunha Jr, R. Nasser, R. Sampaio, H. Lopes and K. Breitman, Uncertainty quantification through Monte Carlo method in a cloud computing setting, Computer Physics Communications (accepted for publication).

7. A. Cunha Jr and R. Sampaio, On the nonlinear stochastic dynamics of a continuous system with discrete attached elements (under review). 